1. Boon, T., and van der Bruggen, P. 1996. Human tumor antigens recognized by $\mathrm{T}$ lymphocytes. J. Exp. Med. 183:725-729.

2. Old, L., and Chen, Y.-T. 1998. New paths in human cancer serology. J. Exp. Med. 187:1163-1167.

3. Diefenbach, A., and Raulet, D. 2002. The innate immune response to tumors and its role in the induction of T-cell immunity. Immunol. Rev. 188:9-21.

4. Dunn, G., Bruce, A., Ikeda, H., Old, L., and Schreiber, R. 2002. Cancer immunoediting: from immunosurveillance to tumor escape. Nat. Immunol. 3:991-998.

5. Mihm, M., Clemente, C., and Cascinelli, N. 1996. Tumor infiltrating lymphocytes in lymph node melanoma metastases: a histopathologic prognostic indicator and an expression of local immune response. Lab. Invest. 74:43-47.
6. Zhang, L., et al. 2003. Intratumoral T cells, recur rence, and survival in epithelial ovarian cancer. N. Eng. J. Med. 348:203-213.

7. Coussens, L., and Werb, Z. 2002. Inflammation and cancer. Nature. 420:860-867.

8. Hanahan, D., and Weinberg, R.A. 2000. The hallmarks of cancer. Cell. 100:57-70.

9. Curcio, C., et al. 2003. Nonredundant roles of antibody, cytokines, and perforin in the eradication of established Her-2/neu carcinomas. J. Clin. Invest. 111:1161-1170. doi:10.1172/ JCI200317426.

10. Pegram, M., et al. 1998. Phase II study of receptorenhanced chemosensitivity using recombinant humanized anti-p185HER2/neu monoclonal antibody plus cisplatin in patients with HER2/neu-overexpressing metastatic breast cancer refractory to chemotherapy treatment. J. Clin. Oncol. 16:2659-2671.
11. Rovero, S., et al. 2000. DNA vaccination against rat her-2/Neu p185 more effectively inhibits carcinogenesis than transplantable carcinomas in transgenic BALB/c mice. J. Immunol. 165:5133-5142.

12. Nanni, P., et al. 2001. Combined allogeneic tumor cell vaccination and systemic interleukin 12 prevents mammary carcinogenesis in HER-2/neu transgenic mice. J. Exp. Med. 194:1195-1205

13. Colombo, M.P., et al. 1991. Granulocyte-colony stimulating factor gene suppresses tumorigenicity of a murine adenocarcinoma in vivo. J. Exp. Med. 173:889-897.

14. Chang, M.-H., et al. 1997. Universal hepatitis B vaccination in Taiwan and the incidence of hepatocellular carcinoma in children. N. Eng. J. Med 336:1855-1859.

15. Koutsky, L., et al. 2002. A controlled trial of a human papillomavirus type 16 vaccine. N. Eng. J. Med. 347:1645-1651.

\title{
The fractalkine receptor CX3CR1 is a key mediator of atherogenesis
}

\author{
Myron I. Cybulsky ${ }^{1}$ and Robert A. Hegele ${ }^{2}$ \\ ${ }^{1}$ Department of Laboratory Medicine and Pathobiology, University of Toronto, \\ Toronto General Research Institute, Toronto, Ontario, Canada \\ ${ }^{2}$ Robarts Research Institute, Blackburn Cardiovascular Genetics Laboratory, \\ London, Ontario, Canada \\ J. Clin. Invest. 111:1118-1120 (2003). doi:10.1172/JCI200318237.
}

Recruitment of circulating monocytes to the arterial intima contributes to the formation of atherosclerotic lesions and may participate in their destabilization. Leukocyte emigration from blood into tissues is mediated by multiple adhesion molecules and chemokines, which orchestrate specific steps of emigration and regulate preferential recruitment of different leukocytes depending on their expression patterns of chemokine receptors. Over the last several years, a number of adhesion molecules, including VCAM-1, P-selectin and ICAM-1, the chemokines MCP-1 (also known as CCL2)

\footnotetext{
Address correspondence to: Myron I. Cybulsky, Department of Laboratory Medicine and Pathobiology, University of Toronto, Toronto General Research Institute, UHN, 200 Elizabeth Street, Eaton-4, Toronto, Ontario, Canada M5G 2C4.

Phone: (416) 340-3578; Fax: (416) 340-3578;

E-mail: myron.cybulsky@utoronto.ca.

Conflict of interest: The authors have

declared that no conflict of interest exists.

Nonstandard abbreviations used: single

nucleotide polymorphism (SNP).
}

and IL-8 (also known as CXCL8), and their respective receptors CCR2 and CXCR2, have been functionally implicated in atherosclerosis. Two studies - one recently published in the JCI (1), and the second reported in this issue (2) - expand this list to include the chemokine receptor CX3CR1, the receptor for fractalkine (also known as CX3CL1).

\section{Fractalkine structure and functions}

Among more than 50 known chemokines, fractalkine is the sole member of the CX3C family, and has unique structural and functional attributes (3, $4)$. In contrast to many other chemokines, whose presentation on the cell surface requires interaction with proteoglycans, the $\mathrm{N}$-terminal chemokine domain of fractalkine is anchored to the cell membrane through a contiguous extended mucin-like stalk, transmembrane and cytoplasmic domains (Figure 1). Fractalkine binding to its seven-transmembrane domain $G$ protein-coupled receptor triggers signaling, but it also directly mediates cell adhesion (5). Fractalkine binds CX3CR1 rapidly and firmly, which leads to tethering and arrest of leukocytes under conditions of physiological flow independent of CX3CR1 signaling (5). TNF- $\alpha-$ converting enzyme (also known as ADAM17) can cleave the mucin stalk of fractalkine and release soluble chemokine $(6,7)$. CX3CR1 has two common coding polymorphisms, namely V249I and T280M, that are in strong linkage disequilibrium (almost always occurring on the same allele) and have been associated with interindividual differences in susceptibility to both HIV infection and atherosclerosis (8-10). If replicated, these findings may have clinical relevance.

\section{The role of CX3CR1 in experimental atherogenesis}

The article by Lesnik et al. (1) demonstrated that fractalkine expression was upregulated in atherosclerotic lesions of apolipoprotein $E^{-/-}\left(\right.$apoe $\left.{ }^{-/-}\right)$mice, primarily in intimal smooth muscle cells, which is consistent with the expression pattern observed previously in human atherosclerosis. The function of CX3CR1 in atherosclerosis was assessed by crossing $\mathrm{CX} 3 \mathrm{CR}^{-/-}$mice into the apoe ${ }^{-/-}$ background and feeding these mice a Western-type diet for 5,10 , or 15 weeks. Lesion formation throughout the aorta, including the aortic root, was significantly reduced in the CX3CR $1^{-1}$ groups. These elegant data provide convincing evidence that CX3CR1 plays an important role in experimental atherogenesis, are consistent with a recent 


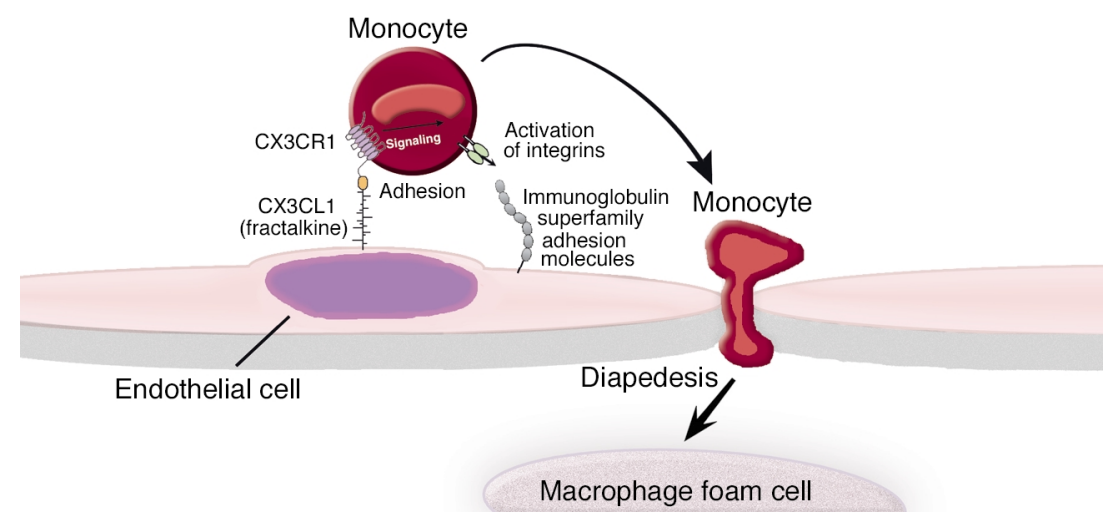

Figure 1

Functions of fractalkine and its receptor CX3CR1 in monocyte recruitment during atherosclerosis. The recruitment of blood monocytes to the arterial intima is one of the earliest events in the formation of an atherosclerotic lesion and persists even in advanced lesions. Monocyte emigration is a multistep process that includes tethering and rolling, arrest, stable adhesion, and diapedesis (transendothelial migration). Shortly after initial tethers are established through binding of selectins or VCAM-1 on activated endothelium, monocytes encounter chemokines bound to endothelial cell-surface proteoglycans. Chemokines initiate signaling via chemokine G protein-coupled receptors, which then activates monocyte integrins and subsequently leads to reorganization of the cytoskeleton. Activated integrins mediate arrest and stable adhesion, and contribute to diapedesis by binding immunoglobulin gene superfamily adhesion molecules, including VCAM-1 and ICAM-1. Fractalkine, like other chemokines, activates multiple intracellular signaling pathways via its receptor. However, it is unique, since it is a transmembrane protein that binds to CX3CR1 rapidly and firmly, which may directly contribute to monocyte tethering and arrest. During atherogenesis, monocytes recruited to the arterial intima transform into macrophages, engulf lipids, and exhibit morphological features of foam cells.

study by Combadiere et al. (11), and establish CX3CR1 as a potential therapeutic target.

In the field of experimental atherosclerosis, sophisticated transgenic and bone marrow transplantation approaches have been used to modulate the expression of candidate genes. However, the analysis, for the most part, consists of simple determination of atherosclerotic lesion area and/or volume and assessment of histological features. Similarly, Lesnik et al. (1) did not provide direct evidence of reduced monocyte recruitment. It is reasonable to assume that deficiency of a chemokine, chemokine receptor, or adhesion molecule will result in decreased monocyte recruitment to the arterial intima, which will translate into reduced lesion area and volume. Yet, one must keep in mind that there may be significant redundancy in molecular pathways contributing to leukocyte recruitment, that expression of adhesion molecules and chemokines is not restricted to the arterial endothelium, and that these molecules have functions outside of
Offspring Cohort, contrasts with most association studies principally because the authors included functional evaluation of the T280M/ V249I polymorphisms. Relative to T280, the atheroprotective M280/ I249 variant when expressed by transfected HEK 293 cells exhibited a lower on-rate of fractalkine binding. Transfected K562 cells or primary leukocytes from homozygous M280/I249 donors revealed markedly decreased adhesive function, signaling (calcium flux), and chemotaxis. These are important and novel insights into the significance of CX3CR1 coding polymorphisms. The association between reduced risk of atherosclerotic cardiovascular disease and CX3CR1-M280/ I249 polymorphisms was based on a dominant model, since the majority of individuals were heterozygotes with a fully functional CX3CR1-T280 allele. Considering that heterozygotes have only a partial deficiency in CX3CR1 function and that redundant pathways for monocyte recruitment may exist, assessment of intermediate phenotypes, such as monocyte recruitment or abundance within lesions, is required to bolster confidence in the idea that the M280/I249 polymorphisms are truly responsible for the significant association with reduced atherosclerotic disease. Other possibilities should be explored. There are several common single nucleotide polymorphisms (SNPs) that lie within the $C X 3 C R 1$ promoter (13) and it is necessary to determine if these affect CX3CR1 transcription and are in linkage disequilibrium with the M280/I249 polymorphisms. Furthermore, as is usually the case in genetic association studies, extra complexity arises from the variability of these linkage relationships among different ethnic groups and population substrata, not to mention the possibility that the observed associations resulted from linkage disequilibrium with unmeasured functional variants in closely linked genes, such as CCR8 (13). Additional studies will be needed to replicate the associations in other samples and the trend in the future should be towards analysis of all possible SNPs at a locus. 


\section{Conclusion}

So where do we stand today? We now appreciate that CX3CR1 and presumably fractalkine are important players in atherogenesis, and that CX3CR1 contains polymorphisms that modulate its adhesive and signaling functions and are associated with reduced risk of atherosclerotic cardiovascular disease. But to truly understand the mechanism of CX3CR1 and other genes in a highly complex and chronic disease process such as atherosclerosis, new practical intermediate markers are required in both experimental models and clinical studies.

1. Lesnik, P., Haskell, C.A., and Charo, I.F. 2003 Decreased atherosclerosis in $\mathrm{CX}_{3} C R 1^{-/-}$mice reveals a role for fractalkine in atherogenesis. J. Clin. Invest. 111:333-340. doi:10.1172/JCI200315555.

2. McDermott, D.H., et al. 2003. Chemokine receptor mutant CX3CR1-M280 has impaired adhesive function and correlates with protection from cardiovascular disease in man. J. Clin. Invest. 111:1241-1250. doi:10.1172/ JCI200316790.

3. Bazan, J.F., et al. 1997. A new class of membranebound chemokine with a CX3C motif. Nature. 385:640-644.

4. Pan, Y., et al. 1997. Neurotactin, a membraneanchored chemokine upregulated in brain inflammation. Nature. 387:611-617.

5. Imai, T., et al. 1997. Identification and molecular characterization of fractalkine receptor CX3CR1, which mediates both leukocyte migration and adhesion. Cell. 91:521-530.

6. Tsou, C.L., Haskell, C.A., and Charo, I.F. 2001. Tumor necrosis factor-alpha-converting enzyme mediates the inducible cleavage of fractalkine. J. Biol. Chem. 276:44622-44626.

7. Garton, K.J., et al. 2001. Tumor necrosis factoralpha-converting enzyme (ADAM17) mediates the cleavage and shedding of fractalkine
(CX3CL1). J. Biol. Chem. 276:37993-38001.

8. Faure, S., et al. 2000. Rapid progression to AIDS in HIV+ individuals with a structural variant of the chemokine receptor CX3CR1. Science 287:2274-2277.

9. McDermott, D.H., et al. 2001. Association between polymorphism in the chemokine receptor CX3CR1 and coronary vascular endothelial dysfunction and atherosclerosis. Circ. Res. 89:401-407.

10. Moatti, D., et al. 2001. Polymorphism in the fractalkine receptor CX3CR1 as a genetic risk factor for coronary artery disease. Blood. 97:1925-1928.

11. Combadiere, C., et al. 2003. Decreased atherosclerotic lesion formation in CX3CR1/apolipoprotein E double knockout mice. Circulation. 107:1009-1016.

12. Hegele, R.A. 2002. SNP judgments and freedom of association. Arterioscler. Thromb. Vasc. Biol. 22:1058-1061

13. DeVries, M.E., et al. 2003. Genomic organization and evolution of the CX3CR1/CCR8 chemokine receptor locus. J. Biol. Chem. 278:11985-11994. doi:10.1074/jcb.M211422200.

\section{RANK ligand and the regulation of skeletal remodeling}

\author{
Norman H. Bell \\ Department of Medicine, Medical University of South Carolina, Charleston, \\ South Carolina, USA
}

J. Clin. Invest. 111:1120-1122 (2003). doi:10.1172/JCI200318358.

It has long been known that estrogen deficiency in animal models and postmenopausal women is associated with increased osteoclastic bone resorption and bone loss (1-5). In the past decade, several important discoveries of some of the key factors involved in osteoclast formation, survival, function, and regulation by estrogen have been made.

A number of hormones and cytokines modulate osteoclastogenesis by enhancing osteoclast differentiation, activation,

Address correspondence to: Norman H. Bell, Department of Medicine, Medical University of South Carolina, 114 Doughty Street, Charleston, South Carolina 29425, USA. Phone: (843) 876-5162; Fax: (843) 876-5163; E-mail: belln@musc.edu.

Conflict of interest: The author has declared that no conflict of interest exists.

Nonstandard abbreviations used: parathyroid hormone (PTH); receptor activator of NF- $\mathrm{KB}$ (RANK); RANK ligand (RANKL); osteoprotegerin (OPG); selective estrogen receptor modulator (SERM). lifespan, and function. These include parathyroid hormone (PTH), calcitriol, PTH-related protein, prostaglandin $\mathrm{E}_{2}$, thyroxine, and IL-11 (3-5). The formation of active osteoclasts requires M-CSF (1,3-6) and involves cell-to-cell contact between precursors of the monocyte/macrophage lineage and osteoblasts, marrow stromal cells, and $\mathrm{T}$ and $B$ cells. These cells express the receptor activator of NF- $\kappa B$ ligand (RANKL), a member of the TNF ligand family, which is essential for this process. RANKL attaches to RANK, a receptor on the cell surface of osteoclasts and osteoclast precursors, to stimulate proliferation and differentiation of cells to form the osteoclast phenotype and inhibit apoptosis. Osteoprotegerin (OPG), a soluble decoy receptor produced by osteoblasts, marrow stromal cells, and other cells, profoundly modifies the effects of RANKL by inhibiting RANKL/ RANK interaction (1, 3-5).
In laboratory animals, estrogen deficiency leads to increased osteoclastogenesis and bone loss, and inhibition of osteoclastogenesis is the major means by which estrogen prevents the loss of bone. This is accomplished by diminishing production of IL-1, IL-6, and TNF- $\alpha-$ cytokines that enhance production of M-CSF and RANKL (1, 3-5) - and downregulating NF-KB and RANKL-induced activation of JNK1 and osteoclastogenic activator protein-1 transcription factors c-Fos and c-Jun (7). Estrogen upregulates OPG (8) and TGF- $\beta$ (9), and TGF- $\beta$ increases OPG expression by osteoblasts and stromal cells (10) and inhibits bone resorption by increasing apoptosis of osteoclasts (11) (Figure 1).

\section{RANKL and postmenopausal bone loss}

Whether the OPG/RANKL/RANK system is involved in bone loss caused by estrogen deficiency in humans was not known until now. In this issue of the JCI (12), Eghbali-Fatourechi and colleagues employ an elegant set of experiments to examine the possible role of RANKL in postmenopausal bone loss. The authors obtained bone marrow mononuclear cells and used surface markers and flow cytometry to isolate and identify preosteoblastic marrow stromal cells, $\mathrm{T}$ lymphocytes, and B lymphocytes in groups of premenopausal women, untreated postmenopausal women, and 